Carnets de géographes

GÉOGRAPHES

Géographie(s) de la lenteur

\title{
Strategies spatiales et gestion de la biodiversité marine
}

Le cas de l'Aire Marine Protégée et Côtière des îles Kuriat en Tunisie

\section{Racha Sallemi}

\section{OpenEdition}

\section{Journals}

Édition électronique

URL : http://journals.openedition.org/cdg/340

DOI : $10.4000 /$ cdg. 340

ISSN : 2107-7266

\section{Éditeur}

UMR 245 - CESSMA

\section{Référence électronique}

Racha Sallemi, «Strategies spatiales et gestion de la biodiversité marine », Carnets de géographes [En ligne], 8| 2015, mis en ligne le 01 septembre 2015, consulté le 24 septembre 2020. URL : http:// journals.openedition.org/cdg/340 ; DOI : https://doi.org/10.4000/cdg.340

Ce document a été généré automatiquement le 24 septembre 2020.

\section{c) (†) $९$}

La revue Carnets de géographes est mise à disposition selon les termes de la Licence Creative Commons Attribution - Pas d'Utilisation Commerciale - Pas de Modification 4.0 International. 


\title{
Strategies spatiales et gestion de la biodiversité marine
}

\author{
Le cas de l'Aire Marine Protégée et Côtière des îles Kuriat en Tunisie
}

\section{Racha Sallemi}

1 Ma thèse concerne l'étude des mécanismes entrant en jeu dans le processus d'acceptabilité et de concrétisation du premier projet d'Aire Marine Protégée (AMP) en Tunisie par rapport à des usages, dont la pêche principalement, mais également l'aquaculture et le tourisme. Cette AMP concerne à la fois un espace marin et un espace insulaire : les îles Kuriat. Ces dernières sont de petites émergences de terre inoccupées sont situées à environ $20 \mathrm{~km}$ du littoral de la ville de Monastir située dans le Sahel tunisien (centre-est). Possédant un statut de protection reconnu à l'échelle méditerranéenne, elles ont fait l'objet d'un plan de gestion car présentant un haut degré de rareté et de vulnérabilité en terme de biodiversité. Les îles Kuriat remplissent ainsi les conditions qui leur permettront d'être éligibles à l'inscription sur la liste des Aires Marines Protégées. S'agissant d'une aire géographique au sein de laquelle les dynamiques humaines sont importantes, j'ai abordé cette problématique à travers le questionnement suivant: en quoi les dynamiques spatiales liées aux usages de la mer au sein de la baie de Monastir peuvent-elles constituer un enjeu dans la perspective de la création d'une AMP aux îles Kuriat? 
Figure 1 : contexte géographique de la baie de Monastir et des îles Kuriat

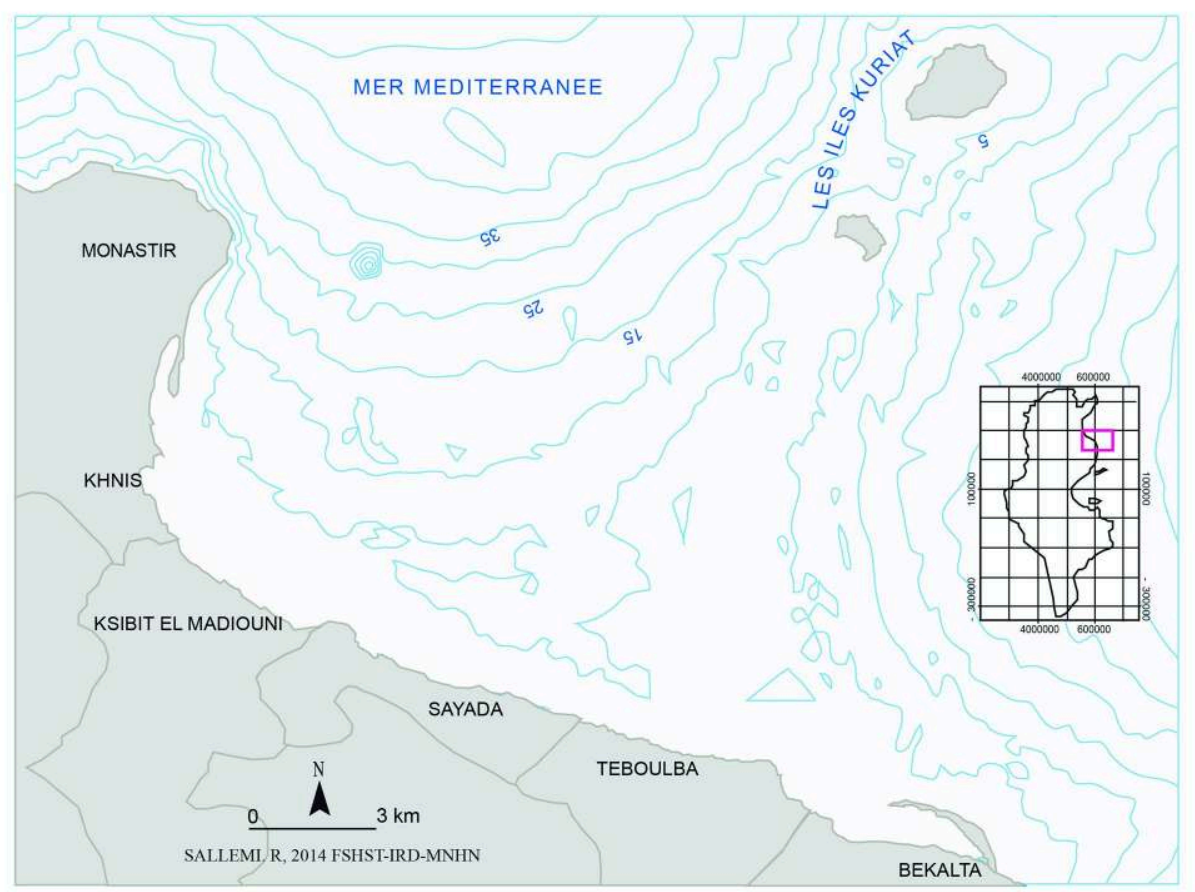

2 Par rapport à ma problématique, trois axes de recherche se rapportant à l'ancrage territorial de l'activité de pêche, à la polarisation halieutique des îles Kuriat, ainsi qu'aux politiques publiques en matière de gouvernance environnementale ont guidé ma thèse.

\section{Un territoire construit à travers l'activité de pêche : l'importance des savoirs locaux}

3 L'analyse historique a permis d'établir comment l'activité de pêche a évolué au fil des siècles et comment elle a marqué le sahel tunisien et la baie de Monastir. Depuis l'époque romaine jusqu'à nos jours, les différentes civilisations qui se sont installées dans cette partie de la Tunisie ont relevé le potentiel halieutique existant et ont axé une partie de leur développement économique sur l'exploitation des ressources marines. L'évolution des techniques de pêche et l'élaboration de méthodes de conservation de la production témoignent de l'importance accordée à la ressource halieutique. Ainsi, au fil des époques et des civilisations, les processus d'accumulation à travers le contact avec les pêcheurs étrangers (italiens et maltais) ont permis aux populations marines de faire valoir un savoir-faire technique et des savoirs naturalistes qui représentent les deux éléments composant un patrimoine local halieutique. L'histoire des îles Kuriat en tant que zone de pêche n'est pas exempte de trace historique comme l'attestent les ruines des usines de salaison et d'un ancien port. En outre, la pêche au thon au moyen d'une madrague aux îles Kuriat atteste depuis des siècles qu'il s'agit d'une zone de passage lors de la migration de cette espèce en Méditerranée. 


\section{Les îles Kuriat : un « bien commun » au centre de stratégies spatiales?}

4 L'analyse du système halieutique appréhendée à travers la typologie en général, la statistique exploratoire et la cartographie des trajectoires de pêche en particulier montre qu'il est possible d'associer plusieurs approches pour tenter de saisir les mécanismes d'un système aussi complexe que la pêche artisanale. Dans le cas de la baie de Monastir, la complexité du milieu social des pêcheurs renforce ainsi le choix d'une analyse se rapprochant le plus possible des comportements des pêcheurs et leurs objectifs de production. Au terme de notre construction typologique de la pêche monastirienne, l'analyse du système halieutique local a révélé l'existence de 12 tactiques de pêche démontrant une grande maitrise de la mer. Ces tactiques ont été définies selon des critères relatifs aux engins de pêche, aux espèces ciblées et aux zones de pêche.

5 Cette maîtrise de l'environnement marin n'est pas chose aisée ; les faits à l'évidence ont révélé qu'il s'agit d'une transmission des savoirs naturalistes en tant que savoir-faire au sein d'une profession qui tend à se développer par filiation. Ce savoir-faire, associé aux capacités opérationnelles et au potentiel de mobilité nous a permis de déterminer quatre grandes classes ou types de pêche : la pêche migrante noble, la pêche d'effort, la pêche démersale mobile et la pêche sédentaire. Ces quatre grandes classes possèdent chacune une stratégie d'exploitation des ressources halieutique et appréhendent par là même leurs propres conceptions de l'Espace halieutique. Si pour les pêches peu ou moyennement mobiles, l'espace halieutique représente l'espace-hérité, celui des pêches très mobiles marque leur indépendance par rapport aux traditions et par rapport à la baie elle-même. Toutefois, les zones de pêche traditionnelles continuent à jouer un rôle polarisateur très important pour une grande majorité de pêcheurs monastiriens quel que soit le type de pêche exercé. À ce titre, les îles Kuriat jouent plusieurs rôles selon la stratégie adoptée. Elles peuvent servir de zone de pêche principale comme chez les types sédentaires et démersaux mobile. En revanche, elles servent de zones de pêche de réserve ou de secours pour la pêche migrante noble et les incursions illégales de certains chalutiers issus de la classe de la pêche d'effort traduisent des comportements prédateurs avec pour objectifs des gains rapides et sans effort.

\section{Le projet d'AMP face aux logiques des acteurs : la divergence?}

Dans la baie de Monastir, l'analyse des jeux d'acteurs a montré que généralement, tout acteur est un usager potentiel tandis que la réciproque n'est pas forcément vraie. Les trois pôles du système d'acteurs que sont les grands pêcheurs (professionnels et récréatifs), les producteurs aquacoles et les promoteurs touristiques se partagent non sans conflits un territoire sensible et fortement menacé de dégradation, la frange littorale n'étant déjà plus un espace d'enjeux tant sa dégradation est avancée. Les équilibres qui prévalaient pendant longtemps ont été perturbés dans un premier temps par l'implantation des projets d'aquaculture, dont le nombre n'a cessé d'augmenter, pour arriver finalement à «fermer la mer » aux petits pêcheurs cantonnés à une petite bande entre la frange littorale polluée et la barrière des cages d'aquaculture. 
Cette situation a contribué à accentuer la pression sur la zone des îles Kuriat comme échappatoire et comme lieu de pêche rentable pour des embarcations faiblement motorisées. Toutefois, la révolution tunisienne est venue perturber les équilibres du pouvoir au niveau de la baie et on assiste à l'émergence des petits pêcheurs locaux, "vierges" de toute connivence avec des élus et des responsables pratiquant du favoritisme. Cela a permis un rééquilibrage et une renégociation des droits d'usage à l'avantage de pêcheurs monastiriens. Dans ce contexte, les îles Kuriat en tant que zones de pêche et de tourisme, commencent à accuser les effets d'une anthropisation en constante évolution. Sa richesse écologique sur terre et sur mer étant reconnue à l'échelle méditerranéenne et nationale, elle constitue dès lors un milieu très convoité. Ce dernier nécessite d'autant plus une protection effective que de la capacité de revendication des usagers s'est vue renforcée après la révolution par rapport à un pouvoir exécutif local affaibli. Ainsi, les pouvoirs publics sont forcés d'abandonner leur posture "sanctuariste " et se réorientent de plus en plus vers l'adoption de concepts tels que la GIZC pour développer des outils plus aptes à être acceptés par des acteurs locaux (syndicat, associations, communes...).

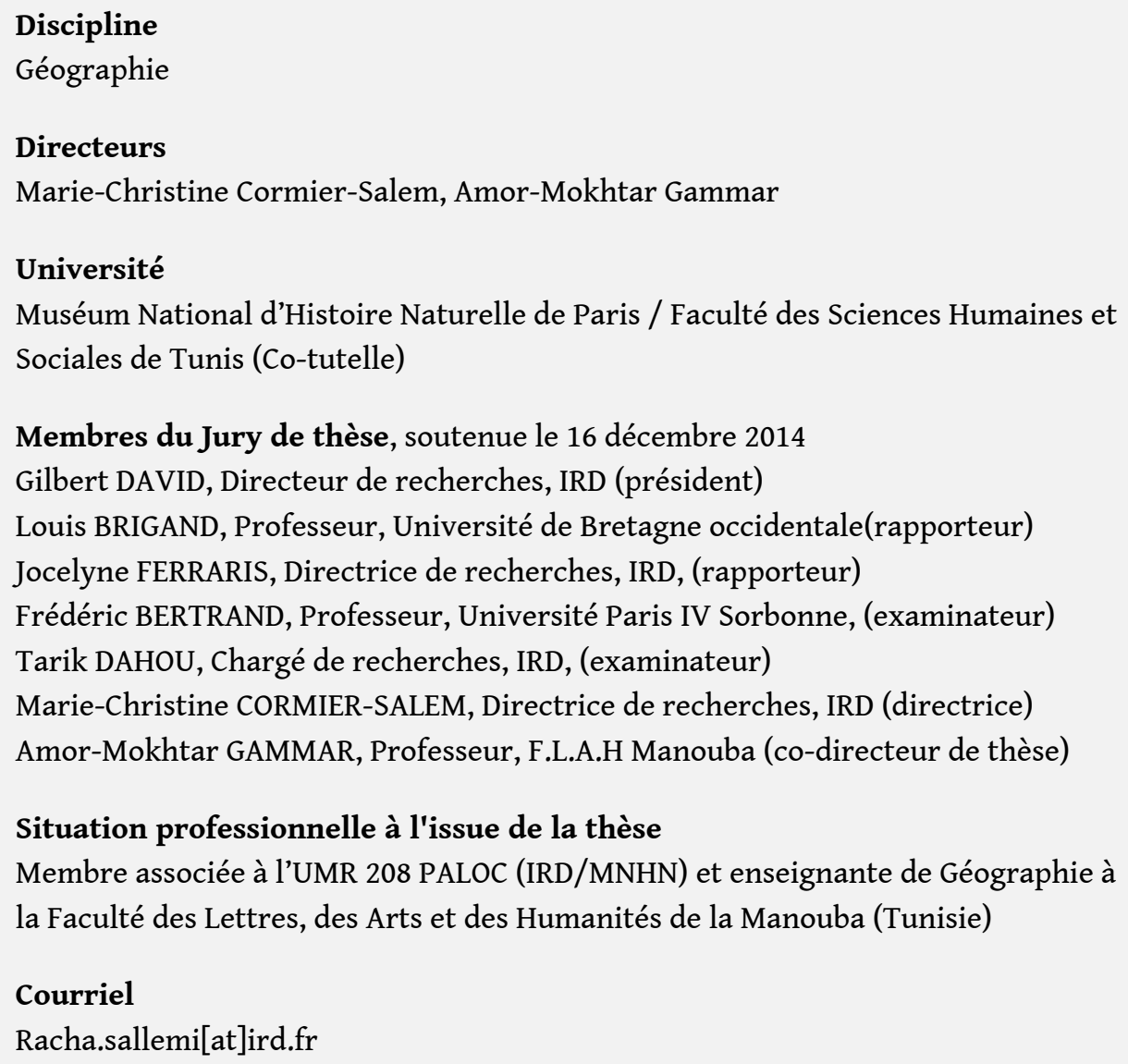


INDEX

Thèmes : Carnets de soutenances 